\title{
Clinico-pathological study of cervical lymphadenopathy at tertiary care centre
}

\author{
Anjana A Mohite ${ }^{1 *}$, Seema S More ${ }^{2,}$ Rajashree Mane ${ }^{3}$
}

\author{
${ }^{1}$ Associate Professor, ${ }^{3}$ Professor \& HOD, Department of ENT and HNS, D.Y.Patil Medical College Hospital and Research Institute, Kolhapur, \\ Maharashtra, INDIA. \\ ${ }^{2}$ Professor and HOD, Dept of Pathology, D.Y.Patil Medical College Hospital and Research Institute, Kolhapur, Maharashtra, INDIA. \\ Email: dranjanamohite@gmail.com
}

\begin{abstract}
Cervical lymphadenopathy is a common presenting complaint of patients attending the ENT out patient department. Enlargement of a lymphnode more than $1 \mathrm{sq} \mathrm{cm}$ indicates a clinical manifestation of a regional or systemic disease serving as an excellent clue to the underlying disease. The differential diagnosis of cervical lymphadenopathy varies from specific and non specific inflammatory conditions to neoplasms and Kikuchi Fujimoto disease. The gold standard biopsy modality in the workup of neck masses is fine-needle aspiration (FNA). The sensitivity and specificity of this cost effective and reliable procedure, in both adults and children has been reported to be approximately $97 \%$ when diagnostic material is obtained. A Prospective Clinico-Pathological study was conducted for two years on 175 patients presenting with cervical lymphadenopathy at D Y Patil Medical College Hospital, a tertiary care centre. Patients more than 12 years both males and females who presented with persistent cervical lymphadenopathy after adequate antibiotic therapy were included in the study while those with acute lymphadenitis and local sepsis were excluded. FNAC was done as primary diagnostic procedure after detailed history taking and clinical examination. The most common diagnosis was Tuberculous lymphadenitis $(54.85 \%)$ followed by Chronic non specific lymphadenitis $(18.28 \%)$, reactive lymphadenitis $(17.14 \%)$, metastatic lymphadenopathy $(8 \%)$. Other diagnosis were Lymphoma $(1.14 \%)$ and Kikuchi Fujimoto disease $(0.57 \%)$. FNAC is one of the most dependable speedy diagnostic tool that also offers a direct approach in the first instance to arrive at a reliable pre operative diagnosis where ever required.

Key Words: Cervical lymphadenopathy, FNAC, Tuberculous lymphadenitis, Non- specific lymphadenitis, Reactive lymphadenitis, Malignant lymphadenitis.
\end{abstract}

*Address for Correspondence:

Dr. Anjana. A. Mohite, 27, Sahajeevan Society, Pachgaon 416013, KOLHAPUR, 416002.

Email: dranjanamohite@gmail.com

Received Date: 05/09/2019 Revised Date: 17/10/2019 Accepted Date: 21/11/2019

DOI: https://doi.org/10.26611/10161231

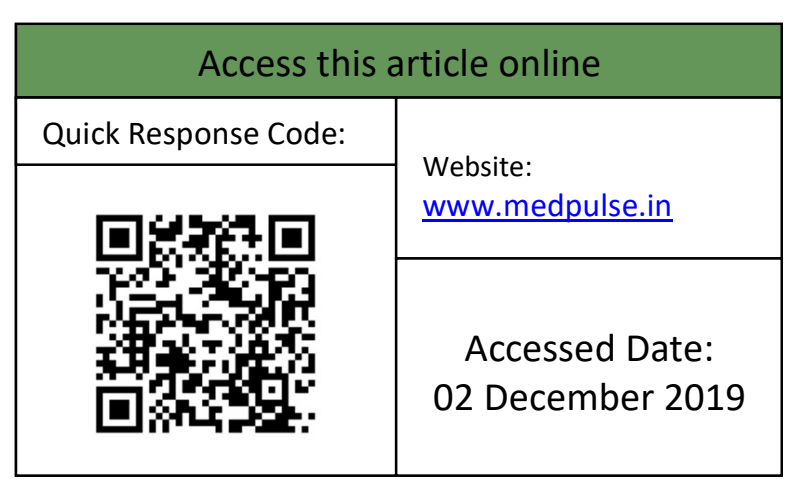

\section{INTRODUCTION}

The cervical lymphnodes account for two thirds of the total lymphnodes of the body. Cervical lymphadenopathy is a common presentation to the clinicians indicating a manifestation of regional or systemic disease. It covers a spectrum of diseases like infections(Specific and non specific), neoplasms, immunodeficiency disorders and Kikuchi Fujimoto disease in its differential diagnosis. Various diagnostic modalities like ultrasonography, CT scan, PET scan and fine needle aspiration cytology (FNAC) are available to diagnose underlying disease in cervical lymphadenitis. All of them have high sensitivity and specificity for cervical lymphadenopathy. The gold standard modality in the workup of neck mass is fine needle aspiration with approximately $97 \%$ sensitivity and specificity when diagnostic aspirate is obtained. FNAC should always be considered before any open procedures. Apart from diagnostic cytology it can also be used for culture in suspected infectious neck masses not responding to conventional antibiotic therapy. If FNAC is unsuccessful or the aspirate is inadequate, repeat FNAC is the protocol before open biopsy. This study was carried out with the broad objective of assessing relative diagnostic efficacy of clinical evaluation and FNAC so 
that a protocol for diagnosis and proper management of cervical lymphadenopathy could be evolved.

\section{MATERIALS AND METHODS}

This prospective study was designed and conducted at D Y Patil Medical College Hospital and Research Centre from March 2017 to February 2019 on 175 patients (OPD and IPD). All patients, males and females more than 12 years who presented with persistent chronic cervical lymphadenopathy were included in the study, while patients with acute infective lymphadenitis and local sepsis were excluded from the study. A written informed consent was taken from all the patients participating in the study. A detailed clinical history was taken with relevance to age, sex, duration of symptoms, constitutional symptoms, contact with tuberculosis and other relevant aspects were noted. This was followed by detailed clinical examination and relevant investigations. All the patients were subjected to FNAC and the reports analysed. The evidence of lymphocytes with epitheliod and Langhan giant cells were reported as tuberculous lymphadenitis. Clustered large cells with nuclear hyperchromasia and pleomorphism were reported as malignancy.

\section{RESULTS}

The present study comprised of 175 cases of chronic cervical lymphadenopathy. The youngest patient was 12 years of age and oldest was 74 years old. Majority of patients affected were in age group of 21 to 30 years followed by 31 to 40 years. Least affected age group was 71 to 80 years. There were 102 males $(58.2 \%)$ and 73 females $(41.7 \%)$ in the study. The male to female ratio in the study was 1.3:1.(Table1)Neck swelling was the presenting complaint in all 175 cases $(100 \%)$, followed by fever in 120 cases $(68.57 \%)$, cough in 36 cases $(20,37 \%)$,

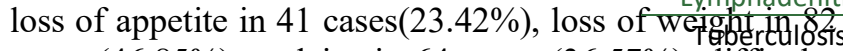
cases $(46.85 \%)$, malaise in 64 cases $(36.57 \%)$, Nofiffigul tiatc in swallowing in 12 cases $(6.85 \%)$ and change in Rearefein 12 cases $(6.85 \%)$. (Table2) On assessing the sitengestafie lymph node involvement, Level I -submetytonlphand submandibular nodes were involved in 30 caskis(4chilf4\%im), oto Level II-upper jugular nodes in 70 cases(40\%), Level IIImiddle jugular nodes in 10 cases(5.71\%), Level IV-lower jugular nodes in 5 cases $(2.85 \%)$, Level V - posterior triangle nodes in 35 cases $(20 \%)$ and more than one site in neck, in 25 cases $(14.30 \%)$. (Table 3 ) In our study out of 175 cases of cervical lymphadenopathy, 159 were non neoplastic and 16 were neoplastic lesions. Of the major causes, Tuberculosis was found to be the most common etiology in 96 cases. (54.85\%) followed by nonspecific lymphadenitis in 32 cases $(18.28 \%)$, reactive lymphadenitis in 30 cases $(17.14 \%)$, and metastatic lymphadenopathy in $14 \operatorname{cases}(8 \%)$. Other causes were
Lymphoma (Hodgkins- 1 and Non Hodgkins-1) in 2 cases $(1.14 \%)$ and Kikuchi Fujimoto disease in 1 case $(0.57 \%)$. (Table 4) In our study the sensitivity of FNAC in diagnosis of Tuberculous lymphadenitis was found to be $82 \%$ and specificity was found to be $100 \%$. (Table 5)

Table 1: Demographic Profile

\begin{tabular}{ccccc}
\hline Age Group(Yrs) & Male & Female & Total & Percentage \\
\hline $12-20$ & 13 & 10 & 23 & 13.14 \\
$21-30$ & 36 & 28 & 64 & 36.59 \\
$31-40$ & 32 & 21 & 53 & 30.28 \\
$41-50$ & 11 & 08 & 19 & 10.85 \\
$>50$ & 10 & 06 & 16 & 9.14 \\
\hline Total & 102 & 73 & 175 & 100 \\
\hline
\end{tabular}

Table 2: Incidence of Symptomatology

\begin{tabular}{ccc}
\hline Symptom & No.of patients & $\%$ \\
\hline Neck swelling & 175 & 100 \\
Fever & 120 & 68.57 \\
Loss of weight & 82 & 46.85 \\
Malaise & 64 & 36.57 \\
Loss of appetite & 41 & 23.42 \\
Cough & 36 & 20.57 \\
Dysphagia & 12 & 6.85 \\
Change in voice & 12 & 6.85 \\
\hline
\end{tabular}

Table 3: Level of Lymphnode Involvement

\begin{tabular}{ccc} 
Level of Lymphnode & No. of Cases & $\%$ \\
\hline I & 30 & 17.14 \\
III & 70 & 40 \\
IV & 10 & 5.71 \\
V & 05 & 2.85 \\
More than 1 site & 35 & 20 \\
\end{tabular}

Table 4: Etiology of Lymphadenopathy with Review of Literature

\begin{tabular}{ccccccc}
\multicolumn{2}{c}{$\begin{array}{c}\text { Our study } \\
\text { No. }\end{array}$} & $\begin{array}{c}\text { Shafiullah } \\
\%\end{array}$ & $\begin{array}{c}\text { Jha B } \\
\%\end{array}$ & $\begin{array}{c}\text { Jindal N } \\
\%\end{array}$ & $\begin{array}{c}\text { Nataraj } \\
\%\end{array}$ & $\begin{array}{c}\text { Arora B } \\
\%\end{array}$ \\
\hline 96 & 54.85 & 69 & 63.8 & 48.4 & 82.6 & 62 \\
32 & 18.28 & 3.8 & 5.9 & 22.8 & 8 & 17 \\
30 & 17.14 & 17.8 & 9.6 & & & \\
14 & 8 & 2.9 & 20.7 & 13.3 & 9.4 & 6 \\
02 & 1.14 & 6.5 & 0 & 15.8 & & 15 \\
01 & 0.57 & 0 & 0 & 0 & 0 & 0 \\
\hline
\end{tabular}

Table 5: Sensitivity and Specificity of FNAC in diagnosis of Tuberculous lymphadenitis

\begin{tabular}{cccc}
\hline \multirow{2}{*}{ FNAC } & \multicolumn{2}{c}{ Tuberculous lymphadenitis } & Total \\
& Positive & Negative & \\
\hline Positive & 75 & 0 & 75 \\
Negative & 21 & 79 & 100 \\
Total & 96 & 79 & 175 \\
\hline
\end{tabular}




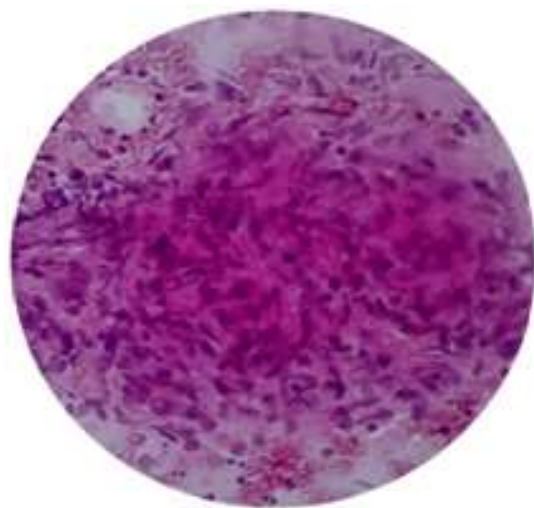

Figure 1: FNAC cervical lymphnode(HandE stain) showing caseous necrosis, epitheliod cells and Langhan giant cells forming Tuberculous granuloma.

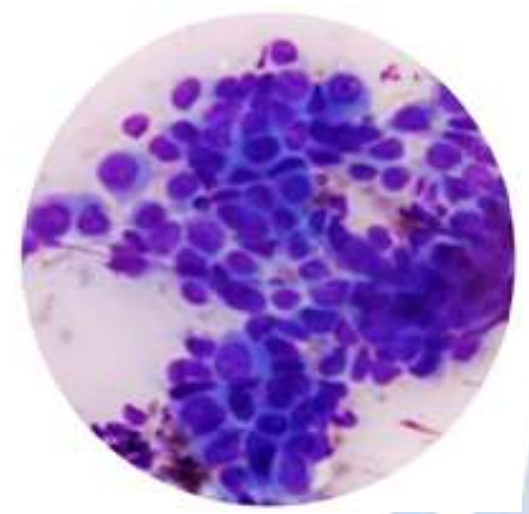

Figure 2: FNAC of lymphnode showing clusters of metastatic tumor cells exhibiting pleomorphism and hyperchromatism

\section{DISCUSSION}

In the present study 175 cases of cervical lymphadenopathy were evaluated to assess the clinicopathological profile and identify various etiologies causing cervical lymphadenopathy. Diagnosis on the basis of FNAC with adequate material for study is almost equally significant as histopathology ${ }^{1}$. FNAC has been an important tool to make a diagnosis in cervical lymphadenopathy cases. We have analysed the data with regards to demography, symptomatology, clinical findings and results of FNAC. The demographic profile showed a male preponderance with mean age in the mid of third decade. In studies by Shafiullah, et al and Kim L $\mathrm{H}$, et $a l^{3}$ the common age groups affected were 20-30 years and 30-40 years respectively. The findings of our study are comparable to those of above mentioned studies. Our study showed a male preponderance which was consistent with studies by Bedi et al , Sarda et al ${ }^{5}$ and Schwarz et al ${ }^{6}$. In our study neck swelling was present in all cases followed by constitutional symptoms. Three fourth of tuberculosis cases and both the lymphoma cases had constitutional symptoms. The same was true in study by Jha B et al ${ }^{7}$.In the present study the assessment of site of lymphnode involved revealed that Level II followed by Level V lymphnodes were most frequent sites of involment. The findings are comparable to the study by Jha B C et al ${ }^{7}$ where the upper jugular group was the most commonly involved and study by Baskota $\mathrm{D}$ et al [8] the posterior triangle nodes were frequently involved. In our study out of 175 cases $90.85 \%$ were non neoplastic whereas $9.15 \%$ were neoplastic lesions. Tuberculous lymphadenitis was diagnosed in $54.85 \%$ of cases followed by non specific lymphadenitis ,reactive lymphadenitis and metastatic lymphadenitis. It was seen in $2^{\text {nd }}$ and 3 rd decade with slight male preponderance presenting clinically as solitary or matted nodes. Cytologically majority showed clusters of epitheliod cells, lymphocytes with or without Langhan cells. The same was true in a study by Paliwal $\mathrm{N}$ et al ${ }^{9}$ Thus tuberculosis was the most common cause for cervical lymphadenopathy in the non neoplastic lesions. It is a very common cause of superficial lymphadenopathy in countries like India. FNAC, even in remote areas of India can be used for diagnosing tuberculous lymphadenopathy with a diagnostic accuracy as high as $100 \%{ }^{10}$. In the neoplastic group metastatic nodes accounted for $8 \%$ and lymphoma accounted for $1.14 \%$. We had 1 case of Kikuchi Fujimoto disease.(0.57\%). The findings were comparable with studies by Haque et al ${ }^{11}$ and Chamyal et al ${ }^{12}$. Not many of the quoted studies have reported Kikuchi Fujimoto disease. We had 1 case in our study. It is an idiopathic necrotizing lymphadenitis first described by Kikuchi in1972. Fujimoto and colleagues independently described the disease in the same year. It is a self limiting disease which may be clinically and histologically mistaken for lymphoma and systemic lupus erythmatosis. One should remain alert to the possibility of Kikuchi Fujimoto disease when a young female presents with cervical lymphadenopathy. Kikuchi Fujimoto disease has a site predilection for cervical lymphnodes and can be diagnosed on FNAC. It must be distinguished from the more common causes of cervical lymphadenopathy like tuberculosis and lymphoma especially in countries like India 13. In the present study, sensitivity and specificity of FNAC in diagnosing tuberculosis was assessed and it was found that sensitivity and specificity of FNAC was $82 \%$ and $100 \%$ respectively. These findings are comparable to studies by Jha B et al, Dandapat M C et al and Mondal A et al ${ }^{14}$.

\section{CONCLUSION}

Tuberculosis, nonspecific lymphadenitis, reactive lymphadenitis and malignancy are the most important causes of cervical lymphadenopathy in our study population. The other rare causes are lymphoma and 
Kikuchi Fujimoto disease. Presentation is in different age groups and with strikingly different clinical features. Meticulous clinical examination should be able to reveal the diagnosis. Fine needle aspiration cytology is extremely sensitive and highly specific investigation for early diagnosis. FNAC, thus can be considered as first line invasive test for any case with cervical lymphadenopathy. The observations in our study may help to formulate a protocol for evaluation of cervical lymphadenopathy cases.

\section{REFERENCES}

1. Koss L G (1994) Diagnostic cytopathology and the histological basis, $4^{\text {th }}$ edn. Lippincott Company, Philadelphia, pp 194-198.

2. Shafiullah S, Shah SH, Rehman A, Arshad H, Norin B. Tuberculosis lymphadenitis in Afgan Refugee. J Ayub Med Coll Abottabad. 2001; 20(2): 463-5.

3. Kim LH, Peh SC, Chan KS, Chai SP. Pattern of lymphnode pathology in private pathology laboratory, Malaysia. J Pathol. 1999; 21(2): 87-93.

4. Bedi RS, Thind GS, Arora VK. A Clinicopathological study of superficial lymphadenopathy in Northern India. Intl J Tub. 1987; 3(4): 189-92.

5. Sarda AK, Bal S, Singh MK, Kapur MM. FNAC as preliminary diagnostic procedure for asymptomatic cervical lymphadenopathy. JAPI. 1990;38(3): 203-5.
6. Schwarz R, Chan NH, MacFarlane JK. FNAC in evaluation of $\mathrm{H}$ and $\mathrm{N}$ masses. Am J Surg 1990;159(5):482-5.

7. Jha BC, Das A, Nagarkar NM, Gupta R, Singhal S. Cervical Tuberculosis Lymphadenopathy: changing clinical pattern and concept in management. BMJ Post graduate Med J. 2000;7(7): 185-7.

8. Baskota DK, Prasad R, Kumar, Sinha B, AmatyaRL. Distribution of lymphnodes in the neck in case of tuberculous lymphadenitis. Acta Otolaryngol. 2004;124(9): 1095-8.

9. Paliwal N, Thakur S, Mullik S , Gupta K. FNAC in Tuberculous lymphadenitis: Experience from a Tertiary level Referral Centre. Indian Journal of Tuberculosis. 2011;58: 102-107.

10. Dandapat MC, Mishra BM, Dash SP, Kar PK. Peripheral lymphnode tuberculosis: a review of 80 cases. Br J Surg, 1990 Aug; 77(8):911-12.

11. Haque MA, Talukder SI. Evaluation of FNAC of lymphnode. Mymensingh Med J. 2003;12(1):33-5.

12. Chamyal PC, Sabarigirish K. Clinico pathological correlation study of cervical lymphnode masses. Int $\mathrm{J}$ Otolaryngol Head Neck Surg. 1997;49(4);404-5.

13. Supari D, Ananthmurthy A. Kikuchi Fujimoto Disease: A study of 24 cases. Indian J Otolaryngol Head and Neck Surg. 2014;66: 69-73:102-107.

14. Mondal A, Mukherjee D, Chatterjee DN. FNAC in diagnosis of cervical lymphadenopathy. J Ind Med Assoc, 1989 Dec;87(2): 281-3.

Source of Support: None Declared Conflict of Interest: None Declared 